\title{
Beta endorphins - molecules of therapeutics
}

\author{
Shrihari T.G®
}

Department of Oral Medicine and Oral Oncology, Krishna Devaraya College of Dental Sciences and Hospital, Karnata, India

\begin{abstract}
Endorphins are endogenous morphine, neuropeptides produced in the pituitary gland. There are three types of endorphins beta-endorphins, enkephalins, and dynorphins. Beta-endorphins are an abundant endorphins synthesized and stored in the anterior pituitary gland; it is a precursor of POMC (Proopiomelanocortin). Betaendorphins has got various mechanisms of actions such as anti-inflammatory activity, analgesic activity, stress buster activity, and immune stimulatory activity can be used to treat various diseases such as chronic inflammatory diseases such as heart disease, Alzheimer's disease, cancer, auto-immune disease, diabetes mellitus, and aging. This article highlights about the basic research findings of beta-endorphins and mechanisms of actions in management of various diseases.
\end{abstract}

Keywords: NF-KB, STAT-3, Cortisol, ACTH, Noradrenaline

$\mathrm{E}$ ndorphins are endogenous morphine, neuropeptides produced in pituitary gland, response to stress and pain. There are three types of endorphins beta endorphins, enkephalins, and dynorphins binds to mu $(\mu)$, kappa $(\mathrm{k})$, and delta (Ò) receptors situated on nervous system and immune cells. Beta-endorphins are an abundant endorphins, more potent than morphine, synthesized and stored in the anterior pituitary gland, it is precursor of POMC (pro-opiomelanocortin). Endorphin receptors are increased during stress such as inflammation, binds abruptly with endorphins.

Holistic is a whole person healing. Human body works as a whole. Instead of considering human body as a whole, if we consider as a parts in treating any disease with reductionist chemical drugs yield better results without adverse effects.

Adverse drug reactions is a major killer in the present world, cancer is a major threat to mankind. Majority of cancers more than $90 \%$ of all cancers are due to external environmental factors such as tobacco, alcohol, and infectious agents (such as HBV, EBV). Human environment is the most important factor in any disease including cancer. Important part of the human environment is human mind; human mind is a human consciousness. Chronic psychological stress is one of the important factors for many diseases including human cancer. Cancer cells works exactly like normal cells. "I do not know any treatment how to kill the cancer cells without killing normal cells" said by novel laureate Albert Szent gyorgi. Advanced cancer treatment modalities such as, surgery, radiotherapy, chemotherapy failed to improve the prognosis of the cancer with increasing morbidity, adverse drug reactions, and decreased survival rate. Endorphins are produced during mindful meditation, massage, pranayama, pranic healing, intense physical exercise creates a psychological relaxed state known as 'Runner's high', love, tender care, music therapy, acupuncture, sympathy and empathy in caring the patient [1-5]. 


\section{MECHANISMS OF ACTIONS OF BETA- ENDORPHIN}

In inflammatory state, recruitment of immune cells to the site of inflammation by chemokine's produces endorphins. Binding of endorphins to the receptors of peripheral nerves results in inhibition of substance $P$, a neuro transmitter of pain and inflammation, produce IL-10, IFNY anti inflammatory cytokines to reduce inflammation. In the PNS, binding of beta endorphin to the $\mu$ receptors situated on the peripheral nerves results in inhibition of substance $P$, a neurotransmitter of pain and inflammation. In the CNS, binding of beta endorphin to the mu receptors situated on the central nervous system results in inhibition of GABA inhibitory neurotransmitter, produce dopamine neurotransmitter involved in analgesic activity, stress buster activity, euphoria, and tranquillity of mind, cognitive development, self reward, and addiction. Endorphin receptors are situated on most innate and adoptive immune cells. Binding of beta endorphins to the mu receptors situated on innate and adoptive cella such as neutrophils, macrophages, mast cells, dendritic cells, natural killer cells, T cells, B cells, results in activation of immune cells(immune stimulatory activity) release opsonin, granzyme-B, interferon $Y$ and antibodies involved in antibacterial activity, antiviral activity, antitumor activity and anti inflammatory activity. Beta endorphins inhibits chronic psychological stress induced sympathetic nervous system activity and activation of parasympathetic nervous system activity of ANS through inhibition of HPA- axis mediated release of neuropeptides such as cortisol, ACTH, and nor adrenaline inhibit inflammatory mediators such as IL-1 $\beta$, IL-6, TNF $\alpha$, and COX2, which inhibits NFKB and STAT3 key transcription factors involved in chronic inflammatory diseases such as heart disease, Alzheimer's disease, cancer, auto immune disease, infectious diseases and diabetes mellitus, aging.

Beta endorphins inhibits chronic psychological stress induced activation of NF-KB a key transcription factor of induced inflammatory mediators involved in conversion of TH1 lymphocytic type to TH2 lymphocytic type, mediated by IL-4, STAT-6 transcription factor, release IL-4, IL-5, IL-13, pro inflammatory cytokines involved in chronic inflammation, tissue damage, immune modulation. Growth factors such as EGF, FGF, VEGF, involved in cell proliferation, cell survival. Altered induced regulatory T cells ( ATregs) formed from TH-1 cells mediated by TGF- $\beta$ inflammatory mediator release IL-2, IL-3, IL-4, IL-5, IL-10, IL-17, pro-inflammatory cytokines involved in immune modulation, otherwise normally regulatory $\mathrm{T}$ cells (nTregs) involved in self-tolerance and immune homeostasis. Proteolytic enzymes such as UPA (Urokinase plasminogen activator), matrix metalloproteases (MMP's) 2,9 involved in tissue damage, all these changes leads to autoimmune diseases. Betaendorphin inhibits chronic psychological stress induced neuropeptide's activate inflammatory mediators such as IL- $1 \beta, \mathrm{TNF} \alpha, \mathrm{COX} 2$ pro-inflammatory cytokines activate NFKB a key transcription factor and IL-6, EGF, FGF, PDGF, pro-inflammatory mediators activates STAT-3 transcription factor, both transcription factors work together involved in cell proliferation by activation of cell cycle regulatory proteins such as cyclin D, E and apoptosis (cell survival) by activation of anti apoptotic proteins such as BCL$\mathrm{XL}, \mathrm{BCL}-2$, angiogenesis by IL-8, COX-2, VEGF, Invasion and metastasis by MMP's 2,9 all these changes lead to tumour progression.

Beta endorphins inhibits chronic inflammatory mediators induced activation of NF-KB a key transcription factor involved in tumour progression, which antagonise P53 tumour suppressor gene, a guardian of the genome mutated in more than $50 \%$ of all cancers by inflammatory mediators such as NO (nitric oxide), ROS, RNS, free radicals, AID (activation induced cytidine deaminase) enzyme expressed by NF-KB a key transcription factor. Beta endorphins express epithelial E-cadherin helps in cell adhesion, loss of epithelial E-cadherin mediated epithelial to mesenchymal transition induced tumour invasion. Beta endorphins delay aging by lengthening telomeres, which otherwise shortened with aging. Another mechanism of delay aging by inhibiting ROS, RNS free radicals from inflammatory cells such as neutrophils, macrophages, dendritic cells during oxidative stress via NADPH oxidase pathway involved in cell aging, tissue damage, DNA damage, gene mutation and cell death (1-9). Beta endorphins are an abundant endorphin useful in natural holistic preventive, therapeutic, health promotive, and palliative treatment of various 
diseases such as heart disease, Alzheimer's disease, cancer, infectious diseases, aging, auto immune disease and diabetes mellitus without adverse effects and inexpensive by its immune stimulatory activity, stress buster activity, analgesic activity and anti -inflammatory activity.

\section{CONCLUSION AND FUTURE PERSPECTIVE}

Endorphins are endogenous morphine, neuropeptides produced in the pituitary gland. Beta-endorphins are an abundant endorphins involved in treatment of various diseases by its analgesic activity, anti-inflammatory activity, stress buster activity, and immune-stimulatory activity without adverse effects and inexpensive. Thorough understanding of betaendorphins, types, mechanisms of actions, duration of action, and prognosis related to disease helpful for future therapeutic purpose.

\section{Conflict of interest}

The author disclosed no conflict of interest during the preparation or publication of this manuscript.

\section{Financing}

The author disclosed that they did not receive any grant during conduction or writing of this study.

\section{REFERENCES}

1. Shrihari TG. Endorphins on cancer: a novel therapeutic approach. J Carcinog Mutagen 2017;8:298.

2. Shrihari TG. Endorphins - A novel hidden magic holistic healer. J Clin Cell Immunol 2018;9:547-52.

3. Shrihari TG. Endorphins - A forgotten hidden magic holistic healer: Minireview. Adv Complement Alt Med 2018;2:170-2. 4. Shrihari TG. Beta-Endorphins - A novel natural holistic healer. J Microb Biochem Technol 2018;10:25-6.

5. IwaszkiewicZ KS, Schneider JJ, Hua S. Targeting peripheral opioid receptors to promote analgesic and anti-inflammatory actions. Front Pharmacol 2013;4:132.

6. Hua S. Neuroimmune interaction in the regulation of peripheral opioid mediated analgesia in inflammation. Front Immunol 2016;7:293.

7. Shrihari TG. Dual role of inflammatory mediators in cancer. Ecancermedicalscience 2017;11:721.

8. Shrihari TG. Quantum healing approach to new generation of holistic healing. Transl Med 2017;7:198.

9. Zhang CQ. Role of Beta-endorphin in control of stress and cancer progression in fetal alcohol exposed rats. Thesis. 2013. doi:10.7282/T3XS5SG3. 